\title{
Synthesis and Characterization of High-Purity Bismuth Nanowires via Seed-Assisted Growth Approach
}

\author{
XIN MU, ${ }^{1}$ WEN-YU ZHAO,${ }^{1,2}$ DAN-QI HE ${ }^{1}$ HONG-YU ZHOU,${ }^{1}$ \\ WAN-TING ZHU, ${ }^{1}$ and QING-JIE ZHANG $^{1,3}$ \\ 1.--State Key Laboratory of Advanced Technology for Materials Synthesis and Processing, Wuhan \\ University of Technology, Wuhan 430070, China. 2.-e-mail: wyzhao@whut.edu.cn. 3.-e-mail: \\ zhangqj@whut.edu.cn
}

\begin{abstract}
Nanowires are considered as high-performance thermoelectric materials with large Seebeck coefficients due to quantum confinement and low thermal conductivity because of enhanced boundary scattering of phonons. In this work, a seed-assisted growth method has been developed to synthesize high-purity bismuth nanowires. The bismuth seeds were first synthesized by reducing $\mathrm{BiCl}_{3}$ in the ice water with $\mathrm{NaBH}_{4}$. The high-purity bismuth nanowires about $40-50 \mathrm{~nm}$ in diameter and several tens of micrometers in length were then grown on bismuth seeds by reducing $\mathrm{NaBiO}_{3}$ with ethylene glycol. X-ray diffraction, scanning electron microscopy, and transmission electron microscopy were employed to characterize the crystal structure, microstructure, and growth direction of the bismuth seeds and nanowires. The effects of temperature, reductant, and bismuth seeds template on the microstructures of the bismuth nanowires were also investigated. The synthesis conditions of bismuth seeds and nanowires were optimized. The selected area electron diffraction pattern confirms that the growth direction of bismuth nanowires is parallel to [1120] direction. It was discovered that high-purity bismuth nanowires with high aspect ratio can be synthesized by precisely controlling the temperature to adjust the nucleation rate of the bismuth nuclei, selecting the appropriate reductant to maintain a low nucleation rate, and using bismuth seeds as the template of the epitaxial growth of the bismuth nuclei.
\end{abstract}

Key words: Bismuth nanowires, seed-assisted growth, technique optimization, structural characterization

\section{INTRODUCTION}

Thermoelectric (TE) materials have attracted extensive attention in the past few decades due to their potential applications in power generation and solid-state cooling. ${ }^{1}$ The performance of TE materials is quantified by the dimensionless figure of merit $Z T$, defined as $Z T=S^{2} \sigma T / \kappa$, where $T, S, \sigma, \kappa$ are the absolute temperature, Seebeck coefficient, electrical conductivity and thermal conductivity, respectively. A good TE material requires high $\sigma$, high $S$, and low $\kappa$. Among various techniques for improving the $Z T$, nanostructure engineering is considered one of the

(Received July 4, 2014; accepted January 20, 2015;

published online February 12, 2015) most effective routes. The $S$ of nanowires can be increased by increasing the density of states near the Fermi level through quantum confinement effects, and the $\kappa$ can be decreased by enhancing the boundary scattering of phonons at the surface and interfaces. ${ }^{2-5}$

Bismuth is a typical and attractive TE material, which has relatively large $\sigma$ due to its high carrier mobility and large carrier mean free path. Dresselhaus $^{2,5}$ predicted that bismuth nanowires had better TE performance than that of bismuth bulk materials. Experimentally, various approaches such as sputtering method, ${ }^{6,7}$ melt spinning method, ${ }^{8}$ vapor deposition process, ${ }^{9,10}$ electrochemical assembly, ${ }^{11}$ stress-induced method, ${ }^{12}$ and template-directed 
synthesis ${ }^{13-17}$ have been employed to synthesize nanowires. A variety of "hard" and "soft" templates for preparing bismuth nanowires have been reported. Anodic alumina membrane ${ }^{13,14}$ or quartz $z^{15,16}$ as "hard" templates and ethylene diamine ${ }^{17}$ as "soft" templates have successfully synthesized bismuth nanowires. However, it is a challenging problem to obtain high-purity bismuth nanowires because it is very difficult to completely remove various "hard" and "soft" templates from the bismuth nanowires products.

To synthesize high-purity bismuth nanowires, we have developed a seed-assisted growth approach by the combination of seed synthesis and seed growth processes. High-purity bismuth nanowires with about $40-50 \mathrm{~nm}$ in diameter and several tens of micrometers in length were successfully synthesized. In our experiment, the bismuth seeds acted as "hard" templates of epitaxial growth of bismuth nuclei. However, there is no need to remove the templates because of the same chemical composition of both the seeds and the nanowires. The measurement of the TE properties of single bismuth nanowire is ongoing; however, it can be predicted that the bismuth seeds do not impact the TE properties of bismuth nanowires because of having the same chemical compositions. The effects of temperature, time, and reductant type on the nucleation rate of bismuth nuclei, the epitaxial growth rate of bismuth nanowires, and microstructures of bismuth seeds and bismuth nanowires have also been investigated in the paper.

\section{EXPERIMENT}

All the reagents, including ethylene glycol (EG, $99 \%)$, bismuth sodium dihydrate $\left(\mathrm{NaBiO}_{3} \cdot 2 \mathrm{H}_{2} \mathrm{O}\right.$, $90 \%)$, bismuth chloride $\left(\mathrm{BiCl}_{3}, 98 \%\right)$, cetyltrimethyl ammonium bromide (CTAB, 99\%), sodium borohydride $\left(\mathrm{NaBH}_{4}, 99 \%\right)$, polyvinylpyrrolidone (PVP, K30), hydrochloric acid (HCl, 36-38\%), and sodium hydroxide $(\mathrm{NaOH}, 96 \%)$ were purchased from Sinopharm Chemical Reagent and used without further purification.

The bismuth nanowires were synthesized by a two-step seed-assisted growth approach composed of the preparation of bismuth seeds and the growth of bismuth nanowires. The synthesis process of the bismuth seeds is as follows: (1) $0.15 \mathrm{~g}$ of CTAB and $11.7 \mathrm{mg}$ of $\mathrm{BiCl}_{3}$ were firstly dissolved in $100 \mathrm{~mL}$ of dilute $\mathrm{HCl}$ to form homogenous reaction solution; (2) the alkaline $\mathrm{NaBH}_{4}$ solution was dropwise added to the reaction solution; and (3) the $\mathrm{NaOH}$ solution was used to adjust the $\mathrm{pH}$ value of the reaction solution. The reaction container was dipped into an ice-water bath to prevent the bismuth seeds from overgrowing and aggregating during the whole synthesis process. The synthesis process of bismuth nanowires is as follows: (1) $1.2 \mathrm{~g}$ of PVP, $0.3 \mathrm{~g}$ of $\mathrm{NaBiO}_{3} \cdot 2 \mathrm{H}_{2} \mathrm{O}$, and $10 \mathrm{~mL}$ of the seed solution were dissolved in $70 \mathrm{~mL}$ of ethylene glycol (EG) to form mixed solution; (2) the mixed solution was stirred and added into a Teflon-lined stainless steel autoclave with a volume of $100 \mathrm{~mL}$; (3) the container was closed and maintained at $413 \mathrm{~K}$ for $24 \mathrm{~h}$; and (4) the bismuth nanowires were centrifuged, washed, and dried under vacuum for $4 \mathrm{~h}$. The theoretical yield of bismuth nanowires in an autoclave with $100 \mathrm{ml}$ is $0.20 \mathrm{~g}$ in our experiment; as a result, about $0.12 \mathrm{~g}$ of bismuth nanowires was obtained. Therefore, the yield of bismuth nanowires is about $60 \%$ in our experimental condition.

The constituent phases of the bismuth nanowires were determined by powder x-ray diffraction (XRD; PANalytical X' Pert PRO) using $\mathrm{Cu} K \alpha$ radiation $(\lambda=0.15418 \mathrm{~nm})$. The microstructures were examined by scanning electron microscopy (SEM; JSM5610LV) and field emission SEM (FESEM; Hitachi S-4800). The crystal structure, microstructures, and the growth directions of the bismuth seeds and nanowires were investigated with TEM images, HRTEM images, and selected area electron diffraction (SAED) pattern under a transmission electron microscope (TEM; JEM-2100F).

\section{RESULTS AND DISCUSSION}

Figure 1 shows the XRD pattern of all the as-prepared samples synthesized by reducing $\mathrm{NaBiO}_{3}$ with EG for $24 \mathrm{~h}$ at different temperatures. It can be seen that all the diffraction peaks for the samples at $413 \mathrm{~K}, 433 \mathrm{~K}$ and $453 \mathrm{~K}$ could be indexed based on the diffraction data of JCPDS 85-1330, indicating that these samples are composed of single-phase bismuth metal. The sample at $393 \mathrm{~K}$ mainly consisted of $\mathrm{Bi}$ and $\mathrm{Bi}(\mathrm{OH})_{3}$.

Figure 2 shows SEM images of the samples synthesized by reducing $\mathrm{NaBiO}_{3}$ with EG for $24 \mathrm{~h}$ at different temperatures. As shown in Fig. 2a, a large amount of $\mathrm{Bi}(\mathrm{OH})_{3}$ and a trace of bismuth nanowires were observed in the sample at $393 \mathrm{~K}$. This indicates that the temperature of $393 \mathrm{~K}$ is too low for the reducing reaction of $\mathrm{NaBiO}_{3}$. The sample at

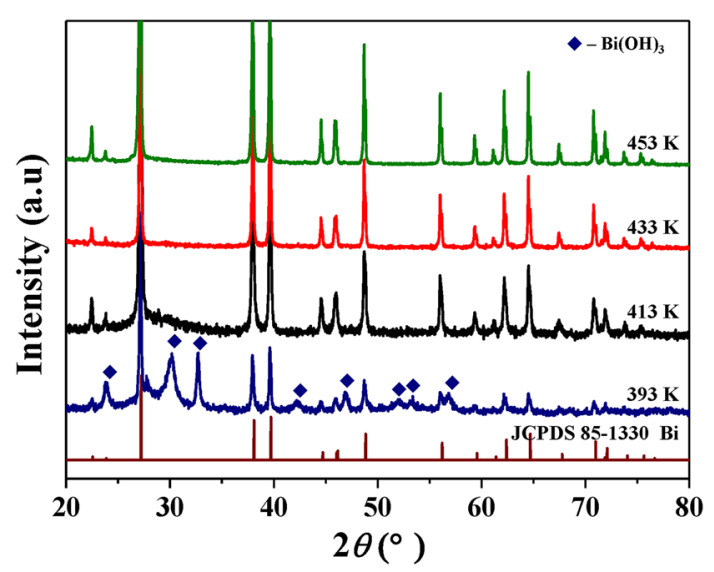

Fig. 1. XRD pattern of the samples synthesized by reducing $\mathrm{NaBiO}_{3}$ with $E G$ for $24 \mathrm{~h}$ at different temperatures. 

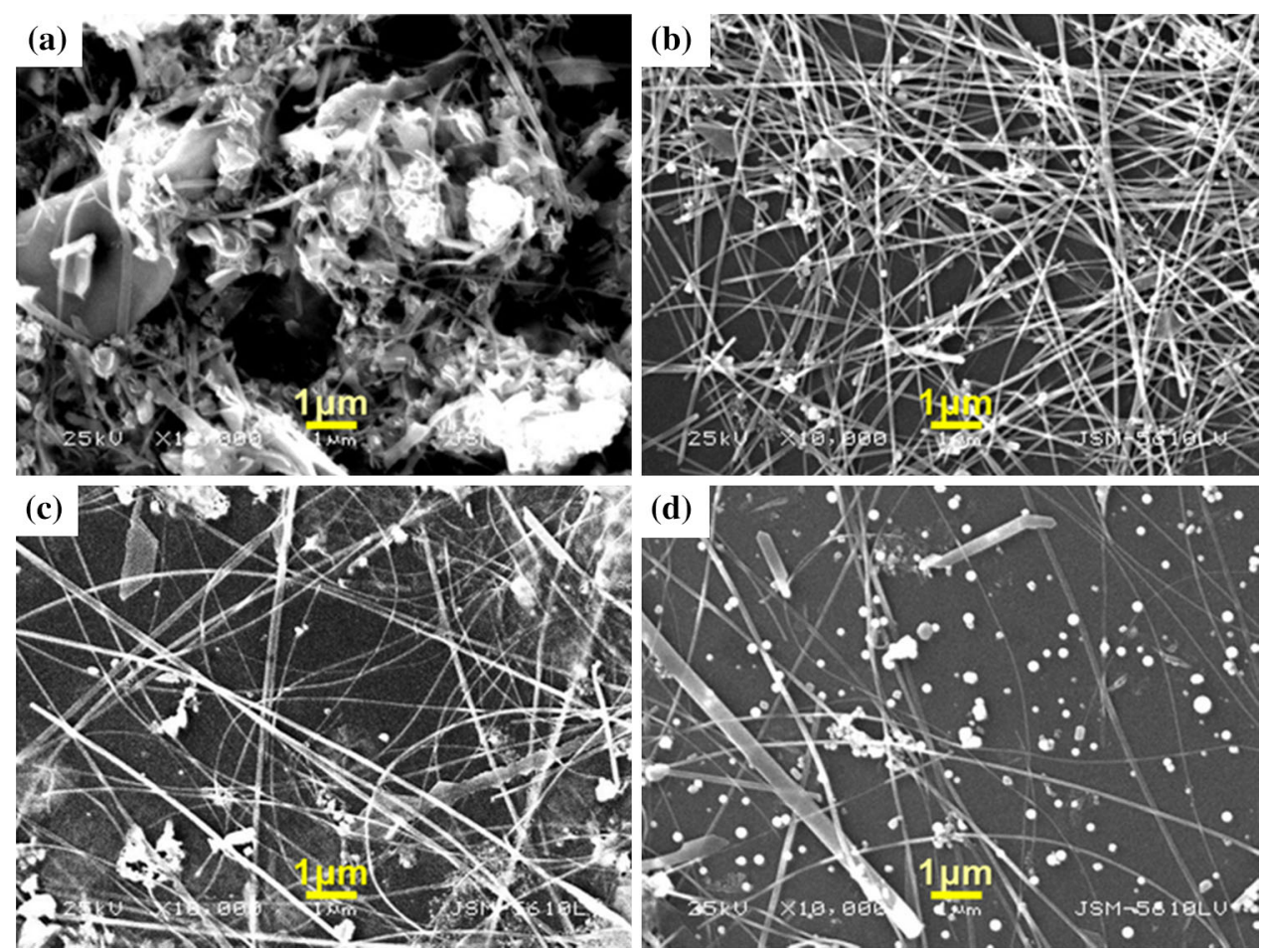

Fig. 2. SEM images of the samples synthesized by reducing $\mathrm{NaBiO}_{3}$ with $\mathrm{EG}$ for $24 \mathrm{~h}$ at different temperatures. (a) $393 \mathrm{~K}$, (b) $413 \mathrm{~K}$, (c) $433 \mathrm{~K}$ and (d) $453 \mathrm{~K}$.

$413 \mathrm{~K}$ is composed of high-purity bismuth nanowires with high aspect ratio, as shown in Fig. 2b. These bismuth nanowires are about $40-50 \mathrm{~nm}$ in diameter and several tens of micrometers in length. The diameters of the nanowires and the number of nanoparticles are gradually increased with increasing the temperature. The diameter of the nanowires reaches about $50-80 \mathrm{~nm}$ in the sample at $433 \mathrm{~K}$, and about $50-100 \mathrm{~nm}$ in the sample at $453 \mathrm{~K}$. In addition, the nanobelts with $300-500 \mathrm{~nm}$ in width are observed in the sample at $453 \mathrm{~K}$. These results indicate that the temperature has important effects in controlling the morphology of the samples. The temperature effects are as follows in this work: (1) increasing the nucleation rate and amount of bismuth nuclei; (2) accelerating the epitaxial growth of bismuth nuclei on the bismuth seeds; (3) providing an advantage for bismuth seeds aggregating into rudiment of bismuth nanowires; and (4) accelerating the bismuth nanowires growth and decreasing the aspect ratio of the bismuth nanowires. However, if the temperature is too high such as up to $453 \mathrm{~K}$, the multi-directional growth will takes place and form nanobelts with $300-500 \mathrm{~nm}$ in width as shown in Fig. 2d. Therefore, by precisely controlling the temperature to adjust the nucleation rate of the bismuth nuclei is an effective approach to synthesize highpurity nanowires with high aspect ratio.

As shown in Fig. 3, both EG and $\mathrm{NaBH}_{4}$ can reduce $\mathrm{NaBiO}_{3}$ into bismuth metal under the conditions of $24 \mathrm{~h}$ and $413 \mathrm{~K}$. However, the products using EG and $\mathrm{NaBH}_{4}$ as reductant have completely different microstructures as shown in Fig. 4. It can be seen that high-purity bismuth nanowires with about 40-60 $\mathrm{nm}$ in diameter are obtained when EG was used as reductant; however, only nanoparticles with about $200-300 \mathrm{~nm}$ in diameter are formed if $\mathrm{NaBH}_{4}$ was used as reductant. These results indicate that the reductant type can greatly affect the morphology of bismuth metal. As is known, the reducibility of EG is much weaker than that of $\mathrm{NaBH}_{4}$. Therefore, the nucleation rate of bismuth nuclei using EG as reductant must be much less than the nucleation rate of bismuth nuclei using $\mathrm{NaBH}_{4}$ as reductant. The formation of bismuth nanowires is attributed to the low nucleation rate of the bismuth nuclei, while the formation of bismuth nanoparticles originates from the high nucleation rate of the nuclei. The low nucleation rate could lead to highly anisotropic growth ${ }^{18}$ and thus assist in fabricating nanowires. In contrast to EG, a low nucleation rate of bismuth nuclei using $\mathrm{NaBH}_{4}$ as reductant was greatly increased and the bismuth nuclei rapidly reached saturation, resulting in the aggregation of bismuth nuclei into bismuth nanoparticles. Therefore, selecting the appropriate reductant such as EG to maintain a low nucleation rate of bismuth nuclei is very important in synthesizing high-purity bismuth nanowires and preventing the formation of bismuth nanoparticles.

Figure 5 shows SEM images of the samples synthesized by reducing $\mathrm{NaBiO}_{3}$ for $24 \mathrm{~h}$ with EG at $413 \mathrm{~K}$ in the presence and absence of bismuth seeds. As shown in Fig. 5a, the high-purity bismuth 
nanowires with about $40-60 \mathrm{~nm}$ in diameter have been successfully synthesized in the presence of bismuth seeds. However, except for the bismuth nanowires, a large number of bismuth nanoparticles of about $40-120 \mathrm{~nm}$ in diameter were synchronously formed in the sample in the absence of bismuth seeds, as shown in Fig. 5b. It is well known that that the seeds used in synthesizing metal nanowires such as $\mathrm{Au},{ }^{19} \mathrm{Ag}^{18,20}$ and $\mathrm{Cu}^{21}$ act as nucleation centers of the reduced metal ions on the seed surfaces and enhance autocatalytic growth. Our experimental results indicate that the bismuth seeds act as template of the epitaxial growth of bismuth nuclei and have an important role in forming the bismuth nanowires and preventing the bismuth nuclei aggregation into nanoparticles. The formation of bismuth nanowires without the bismuth seeds resembled a "self-seeding" process suggested by Sun et al. ${ }^{22}$ It is easy to understand that early formed bismuth nuclei might also act as the centers for the epitaxial growth of later formed bismuth nuclei, forming bismuth nanowires during the "self-seeding" process, though some bismuth nuclei were aggregated and formed the nanoparticles in the sample synthesized without the bismuth

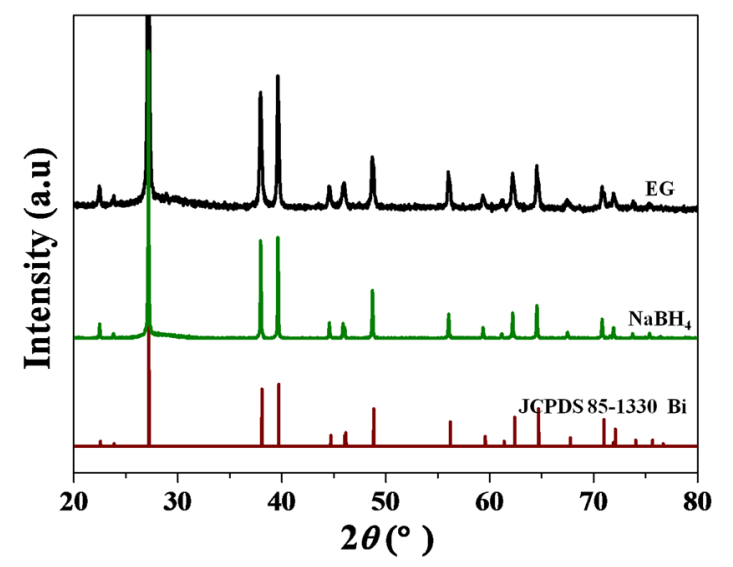

Fig. 3. XRD pattern of the samples synthesized by reducing $\mathrm{NaBiO}_{3}$ with $\mathrm{EG}$ and $\mathrm{NaBH}_{4}$ for $24 \mathrm{~h}$ at $413 \mathrm{~K}$. seeds as template. Therefore, using the bismuth seeds as template is very important to synthesize high-purity bismuth nanowires because the bismuth seeds in fact provide the centers for the epitaxial growth of bismuth nuclei.

Figure 6 shows TEM images and SAED pattern of bismuth seeds. The background image originated from organic CTAB. The bismuth seeds were spherical and about $5 \mathrm{~nm}$ in diameter. The lattice spacing is about $0.1929 \mathrm{~nm}$, corresponding to that of (0006) planes of the hexagonal bismuth (JCPDS 851330). The SAED pattern of the bismuth seeds is a typical polycrystalline diffraction pattern, and the diffraction spots are distributed on several diffraction rings. The lattice spacings of these diffraction spots were calculated and assigned to the (0003),

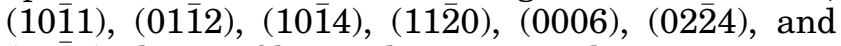
(1126) planes of bismuth, respectively.

Figure 7 displays TEM images (a, b), HRTEM image (c), and SAED pattern (d) of a bismuth nanowire of about $50 \mathrm{~nm}$ in diameter. It can be seen that the nanowires are single-crystalline and defectfree. As shown in Fig. 7c, the lattice spacing is about $0.326 \mathrm{~nm}$, corresponding to those of (1012) and $(01 \overline{12})$ planes of bismuth metal. The inclination angle of the two planes to the bismuth nanowire is about $97.8^{\circ}$. SAED pattern of a bismuth nanowire as shown in Fig. $7 d$ reveals that the nanowire was single-crystalline and the diffraction spots were indexed, indicating that the growth direction of bismuth nanowires is parallel to [1120] direction.

The formation of bismuth nanowires involved two processes. In the first process, bismuth seeds of about $5 \mathrm{~nm}$ in diameter were synthesized by reducing $\mathrm{BiCl}_{3}$ with $\mathrm{NaBH}_{4}$ in organic aqueous CTAB:

$$
\begin{aligned}
& 8 \mathrm{BiCl}_{3}+3 \mathrm{NaBH}_{4}+24 \mathrm{NaOH}=8 \mathrm{Bi}+3 \mathrm{NaBO}_{2} \\
& \quad+18 \mathrm{H}_{2} \mathrm{O}+24 \mathrm{NaCl}
\end{aligned}
$$

$\mathrm{NaBH}_{4}$ with strong reducibility was used to obtain a high nucleation rate of bismuth nuclei and small bismuth seeds with very narrow size distribution. It was very important to prevent the bismuth
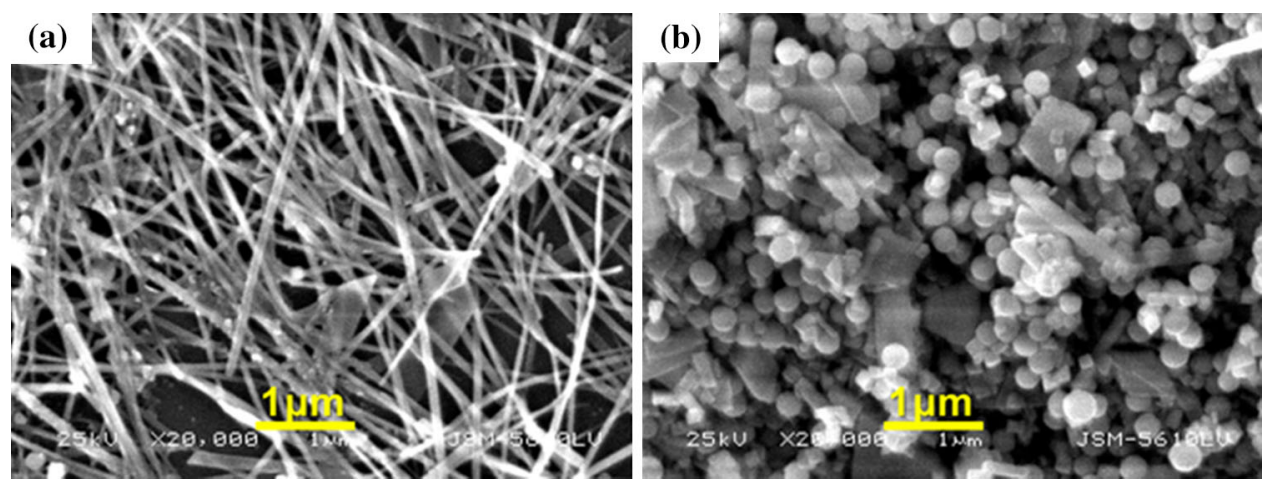

Fig. 4. SEM images of the samples synthesized by reducing $\mathrm{NaBiO}_{3}$ with (a) $\mathrm{EG}$ and (b) $\mathrm{NaBH}_{4}$ for $24 \mathrm{~h}$ at $413 \mathrm{~K}$. 

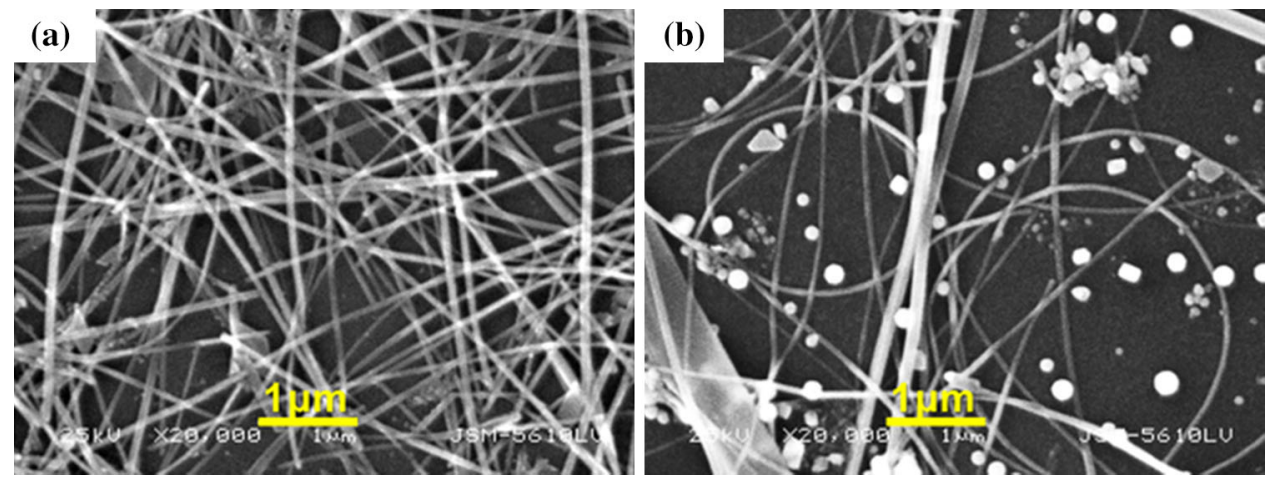

Fig. 5. SEM images of the samples synthesized by reducing $\mathrm{NaBiO}_{3}$ for $24 \mathrm{~h}$ with $\mathrm{EG}$ at $413 \mathrm{~K}$ in the (a) presence and (b) absence of bismuth seeds.
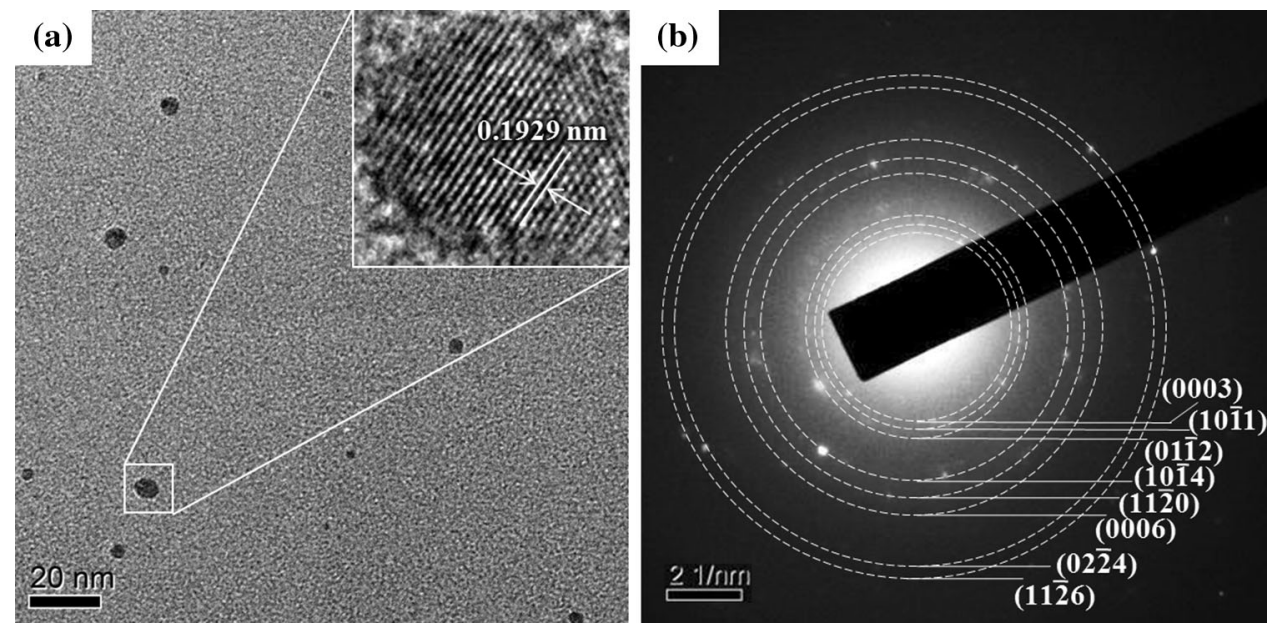

Fig. 6. TEM image (a) and SAED pattern (b) of bismuth seeds. The inset in (a) shows HRTEM image of a bismuth seed.

nuclei from aggregating into big bismuth nanoparticles in our experiment. Therefore, the reaction container had to be dipped into an ice-water bath in the whole process. In addition, organic CTAB was also used as the dispersant to reduce the surface tension bismuth crystal and the aggregation of bismuth seeds.

In the second process, the bismuth seeds served as "hard" templates of epitaxial growth of the bismuth nuclei. The microstructures of the products synthesized by reducing $\mathrm{NaBiO}_{3}$ with EG at $413 \mathrm{~K}$ for different times were investigated to find out how the bismuth seeds grew into bismuth nanowires. Figure 8 displays (a) TEM image of bismuth seeds and (b-e) SEM images of the samples synthesized by reducing $\mathrm{NaBiO}_{3}$ with $\mathrm{EG}$ at $413 \mathrm{~K}$ for (a) $0 \mathrm{~h}$, (b) $0.5 \mathrm{~h}$, (c) $1 \mathrm{~h}$, (d) $4 \mathrm{~h}$, and (e) $24 \mathrm{~h}$. It can be seen that the bismuth seeds of about $5 \mathrm{~nm}$ in diameter as shown in Fig. 8a grew into monodispersed bismuth nanoparticles with about $30-50 \mathrm{~nm}$ in diameter after half an hour as shown in Fig. 8b. After an hour, the monodispersed bismuth nanoparticles had been agglomerated into nanoclusters, as shown in Fig. 8c. The nanoclusters were transformed into short nanorods of about $50 \mathrm{~nm}$ in diameter by increasing the reaction time as shown in Fig. 8d. The nanorods were lastly transformed into nanowires of about $50 \mathrm{~nm}$ in diameter as shown in Fig. 8e.

The morphological evolution with time may be described by a mechanism as sketched in Fig. 9 . Namely, the bismuth seeds were firstly transformed into bismuth nanoparticles by the epitaxial growth of bismuth nuclei on the bismuth seeds, then agglomerated into nanoclusters and lastly into nanorods and nanowires. The smooth surface of the nanorods and nanowires indicates that the selfhealing effect may have an important role in minimizing the surface area and reducing the surface tension. Several bismuth nanorods were concatenated end to end into long nanorods. The concatenated long nanorods were further rearranged into linear bismuth nanowires. The chemical reaction is as follows during the formation of bismuth nanowires:

$$
\begin{aligned}
& 2 \mathrm{NaBiO}_{3}+5\left(\mathrm{CH}_{2} \mathrm{OH}\right)_{2}=2 \mathrm{Bi}+2 \mathrm{CH}_{3} \mathrm{COONa} \\
& \quad+3 \mathrm{CH}_{3} \mathrm{COOH}+6 \mathrm{H}_{2} \mathrm{O} .
\end{aligned}
$$



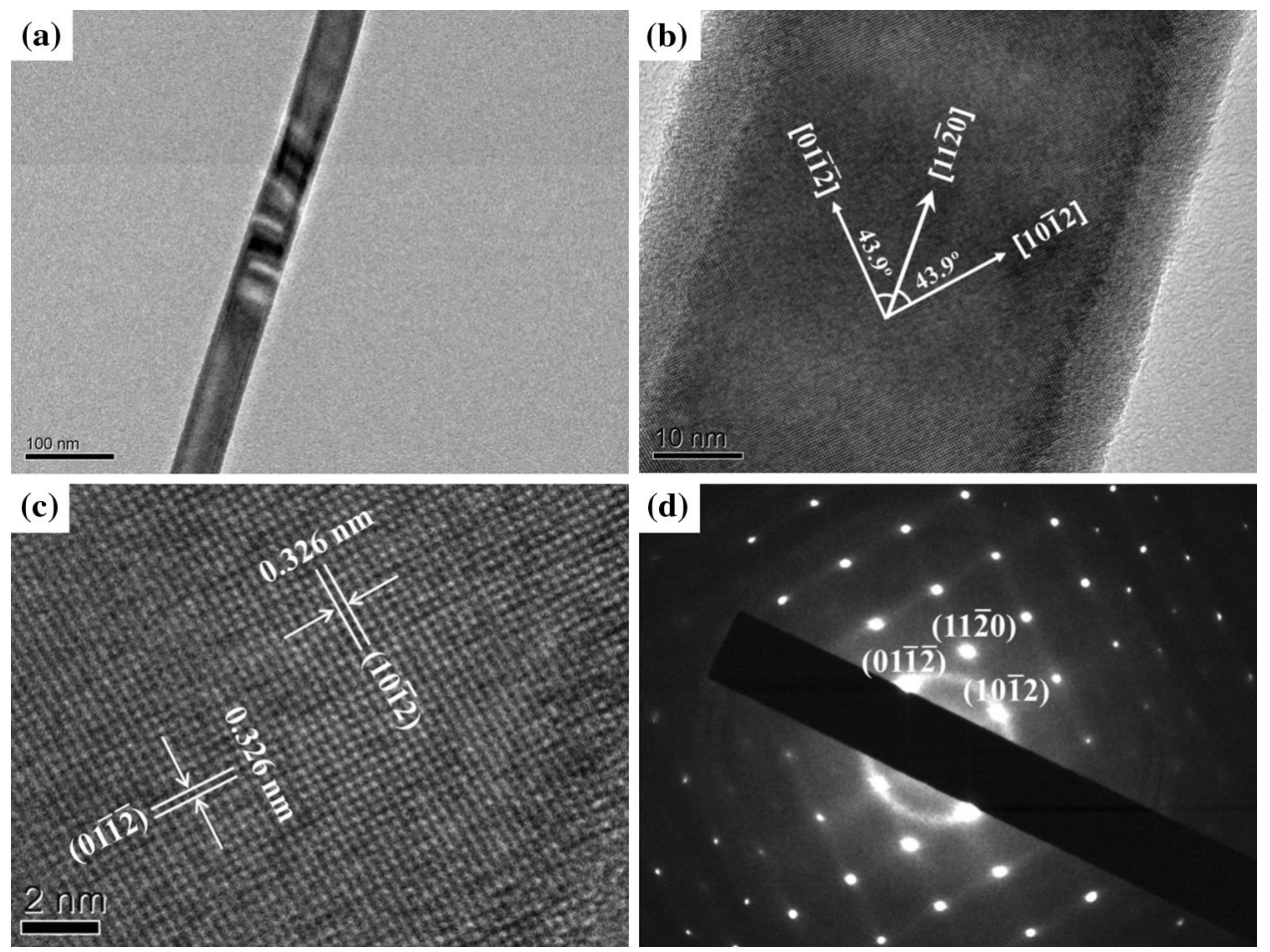

Fig. 7. TEM images (a, b), HRTEM image (c) and SAED pattern (d) of a bismuth nanowire.
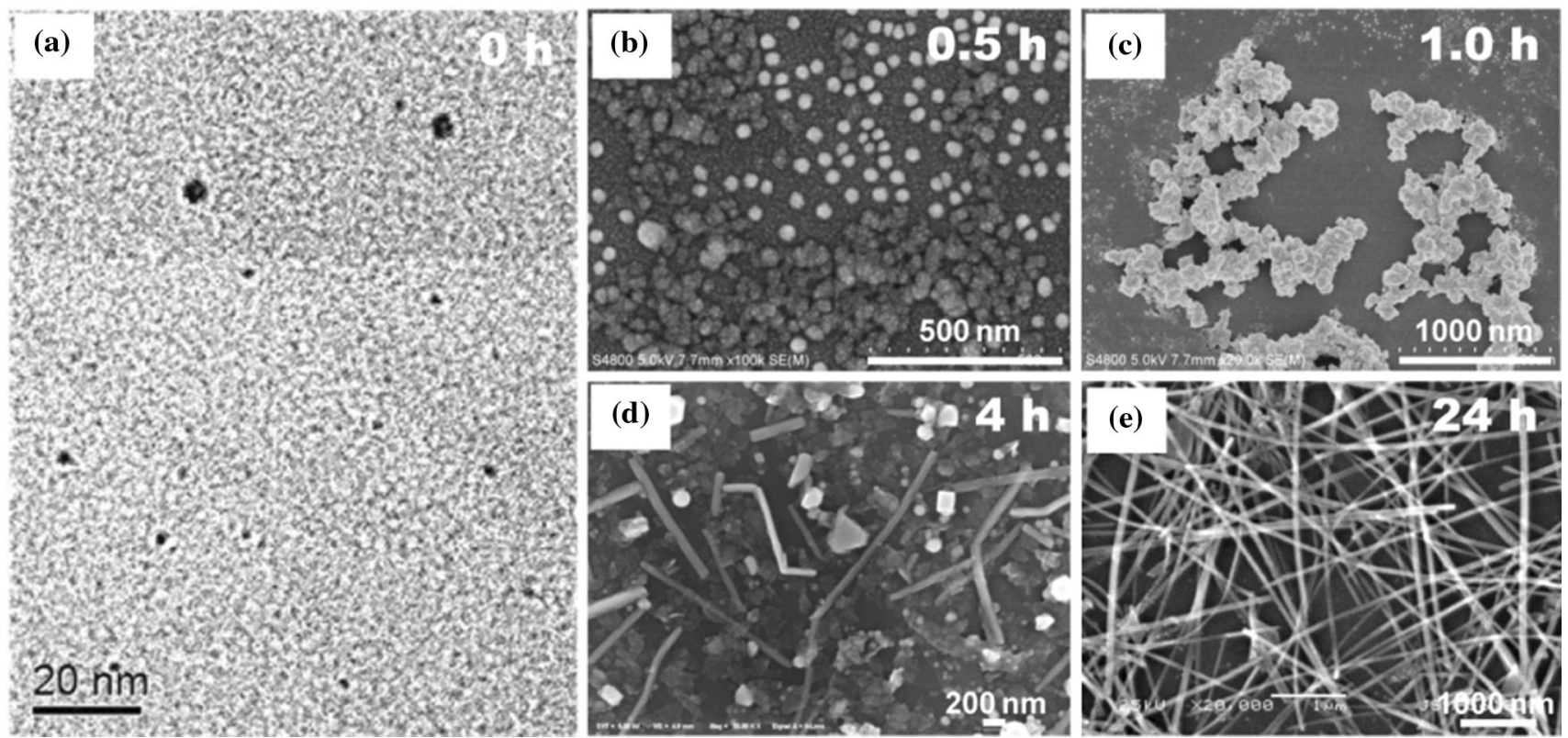

Fig. 8. (a) TEM image of bismuth seeds and (b-e) SEM images of the samples synthesized by reducing $\mathrm{NaBiO}_{3}$ with $\mathrm{EG}$ at $413 \mathrm{~K}$ for (a) $0 \mathrm{~h}$, (b) $0.5 \mathrm{~h}$, (c) $1 \mathrm{~h}$, (d) $4 \mathrm{~h}$, and (e) $24 \mathrm{~h}$.

The high-purity bismuth nanowires have been successfully synthesized because the bismuth seeds template provided the centers of epitaxial growth of bismuth nanowires. To prevent the nanowires from aggregating, the surfactant PVP was used to chemically adsorb onto the surfaces of bismuth nanowires by $\mathrm{O}-\mathrm{Bi}$ bonding. ${ }^{23}$ Many reports ${ }^{18,24,25}$ have indicated that PVP had the functions of controlling the growth rates of different crystalline faces and assisting in fabricating the nanowires. However, our work indicates that both nanoparticles and nanowires had been synchronously formed in the assistance of PVP in the lack of bismuth seeds. Therefore, it is very necessary to use bismuth 


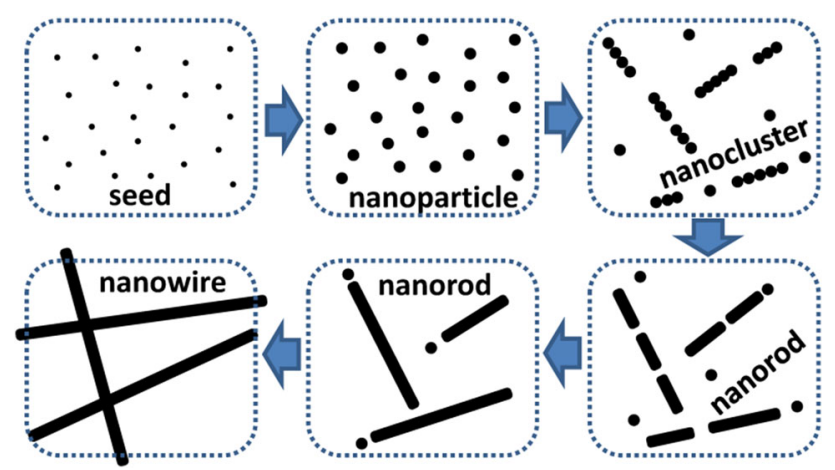

Fig. 9. A plausible mechanism for the formation process from bismuth seeds to nanoparticles to nanoclusters to nanorods to nanowires.

seeds as the template of epitaxial growth of bismuth nanowires for obtaining high-purity bismuth nanowires and preventing the formation of bismuth nanoparticles. In addition, we have also investigated the effects of the volume of the seeds solution, surfactant type, and concentration of $\mathrm{NaBiO}_{3}$ solution on the formation of bismuth nanowires. We found that the volume of seeds solution from $8 \mathrm{ml}$ to $12 \mathrm{ml}$ seemed to have no significant effect on the morphology of the bismuth nanowires, and further work is ongoing to obtain an optimal volume of the seeds solution. Some experimental results on surfactant type and the concentration of $\mathrm{NaBiO}_{3}$ solution are similar to the results reported in the Refs. 17, 23, and 26. In addition, the latest experiments indicate that the effects of other conditions such as $\mathrm{pH}$ value and magnetic field strength can manipulate the kinetics of bismuth nanowires as reported by some researchers. ${ }^{27,28}$

\section{CONCLUSION}

In the present work, a seed-assisted growth approach has been developed to synthesize highpurity bismuth nanowires with high aspect ratio. The bismuth nanowires are about $40-50 \mathrm{~nm}$ in diameter and several tens of micrometers in length. The growth direction of bismuth nanowires has been confirmed to be parallel to the [1120] direction. Three kinds of controlling techniques have been discovered in the synthesis process of high-purity bismuth nanowires and are as follows: (1) by controlling the temperature to precisely adjust the nucleation rate of bismuth nuclei, the optimized temperature is about $413 \mathrm{~K}$; (2) by selecting an appropriate reductant to maintain a low nucleation rate of bismuth nuclei, the suitable reductant for synthesizing high-purity bismuth nanowires is EG at $413 \mathrm{~K}$; and (3) by providing the centers for the epitaxial growth of bismuth nuclei using bismuth seeds as the "hard" template. A plausible mechanism from bismuth seeds to nanoparticles to nanoclusters to nanorods to nanowires is suggested to describe the formation process of bismuth nanowires.

\section{ACKNOWLEDGEMENTS}

This work was supported by the National Basic Research Program of China (973-program) under Project No. 2013CB632505 and National Natural Science Foundation of China (No. 11274248).

\section{REFERENCES}

1. L.E. Bell, Science 321, 1457 (2008).

2. M.S. Dresselhaus, G. Dresselhaus, X. Sun, Z. Zhang, S.B. Cronin, and T. Koga, Phys. Solid State 41, 679 (1999).

3. Y.M. Lin and M. Dresselhaus, Phys. Rev. B 68, 75304 (2003).

4. M.L. Tian, J. Wang, Q. Zhang, N. Kumar, T.E. Mallouk, and M.H.W. Chan, Nano Lett. 9, 3196 (2009).

5. M.S. Dresselhaus, Z. Zhang, X. Sun, J.Y. Yingc, J. Heremanse, G. Dresselhaus, and G. Chen, Mat. Res. Soc. Symp. Proc. 545, 215 (1999).

6. B.K. Wu, H.Y. Lee, and M.Y. Chern, Appl. Phys. Express 6, 35504 (2013).

7. J. Ham, W. Shim, D.H. Kim, K.H. Oh, P.W. Voorhees, and W. Lee, Appl. Phys. Lett. 98, 0431024 (2011).

8. E. Condrea, A. Nicorici, A. Gilewski, and S. Matyjasik, J. Low Temp. Phys. 174, 232 (2014).

9. S. Iyengar, D. Liang, X. Gao, and A.R. Abramson, Acta Mater. 60, 2369 (2012).

10. H.L. Zhang, W. Chen, X.S. Wang, J. Yuhara, and A.T.S. Wee, Appl. Surf. Sci. 256, 460 (2009).

11. A. Nerowski, J. Opitz, L. Baraban, and G. Cuniberti, Nano Res. 6, 303 (2013).

12. W. Shim, D. Kim, K. Lee, K.J. Jeon, J. Ham, J. Chang, S. Han, W.Y. Jeung, M. Johnson, and W. Lee, J. Appl. Phys. 104, 073715 (2008).

13. X.F. Wang, J. Zhang, H.Z. Shi, Y.W. Wang, G.W. Meng, X.S. Peng, L.D. Zhang, and J. Fang, J. Appl. Phys. 89, 3847 (2001).

14. J. Heremans, C.M. Thrush, Y. Lin, S. Cronin, Z. Zhang, M.S. Dresselhaus, and J.F. Mansfield, Phys. Rev. B 61, 2921 (2000).

15. M. Murata, Y. Hasegawa, T. Komine, and T. Kobayashi, Nanoscale Res. Lett. 7, 505 (2012).

16. D. Nakamura, M. Murata, Y. Hasegawa, T. Komine, D. Uematsu, S. Nakamura, and T. Taguchi, J. Electron. Mater. 39, 1960 (2010).

17. Y.H. Gao, H.L. Niu, C. Zeng, and Q.W. Chen, Chem. Phys. Lett. 367, 141 (2003).

18. Y. Sun, Y. Yin, B.T. Mayers, T. Herricks, and Y. Xia, Chem. Mater. 14, 4736 (2002).

19. Y.N. Wang, W.T. Wei, C.W. Yang, and M.H. Huang, Langmuir 29, 10491 (2013).

20. C. Preston, Y.L. Xu, X.G. Han, J.N. Munday, and L.B. Hu, Nano Res. 6, 461 (2013).

21. S.R. Ye, A.R. Rathmell, Y.C. Ha, A.R. Wilson, and B.J. Wiley, Small 10, 1771 (2014).

22. Y. Sun and X. Y, Adv. Mater. 14, 833 (2002).

23. J.W. Wang, X. Wang, C. Peng, and Y.D. Li, Inorg. Chem. 43, 7552 (2004).

24. H.S. Qian, S.H. Yu, J.Y. Gong, L.B. Luo, and L.F. Fei, Langmuir 22, 3830 (2006).

25. K. Wang, H.W. Liang, W.T. Yao, and S.H. Yu, J. Mater. Chem. 21, 15057 (2011).

26. X.Y. Liu, J.H. Zeng, S.Y. Zhang, R.B. Zheng, X.M. Liu, and Y.T. Qian, Chem. Phys. Lett. 374, 348 (2003).

27. Y. Wang and K.S. Kim, Nanotechnology 19, 265303 (2008).

28. Y. Xu, Z. Ren, W. Ren, G. Cao, K. Deng, and Y. Zhong, Nanotechnology 19, 115602 (2008). 